\title{
Changing Student Conceptions of Newton's Laws Using Interactive Video Vignettes
}

\author{
Jonathan Engelman ${ }^{1}$ and Kathy Koenig ${ }^{2}$ \\ ${ }^{I}$ Science and Mathematics Department, Kettering College, 3737 Southern Boulevard, Kettering, Ohio, 45429 \\ ${ }^{2}$ Physics Department, University of Cincinnati, 345 Clifton Court, Cincinnati, Ohio, 45221
}

\begin{abstract}
Changing students' incorrect conceptions in physics is a difficult process and has been studied for many years. Using a framework of elicit, confront, resolve, and reflect, this study analyzed student experiences with two Interactive Video Vignettes designed to change student misconceptions regarding Newton's Second and Third Laws using a fully integrated mixed methods design. This paper explores how differences in how students experienced the framework impacted how much they were able to change their inaccurate conceptions of Newton's Laws.
\end{abstract}

\section{INTRODUCTION}

Changing student conceptions in physics is inherently challenging [1]. Alternative conceptions and conceptual change have been widely studied across many topics in physics [2-5], and results from the literature suggest that students may continue to exhibit many alternative conceptions even after instruction [5]. Although multiple curricula and teaching strategies have been developed and shown to be effective [6], less is known about how these ideas might be effectively integrated into web-based instruction. This becomes an important area of study as student access to technology has increased and more instructors have adopted active learning strategies in their classrooms, placing greater expectations on students to learn core concepts outside of class.

\section{LITERATURE AND STUDY BACKGROUND}

One form of web-based instruction includes the use of Interactive Video Vignettes (IVVs) created by the Live Photo Physics Group [7]. IVVs are designed to supplement classroom face-to-face instruction and center around a single topic, typically one which involves known alternative conceptions reported in the literature [7]. They combine segments of video interspersed with moments of user interaction such as graphing activities or multiple choice questions. Each IVV takes between 4 and 10 minutes to complete. Nine IVVs are currently available for topics including Newton's three laws as well as several other physics topics [8].

Although the IVVs were developed based on findings of physics education research, as well as the work of others who have created web-based instruction involving video (see Derek Muller's Veritasium on YouTube), there are multiple instructional challenges that are difficult to overcome. Two of these include the absence of an instructor who can immediately engage students in Socratic dialogue when misconceptions are identified, as well as the opportunity for students to learn through discussion with peers. Muller [9] has addressed the latter by including interviews with persons on the street in his videos. These serve to generate discussion of alternate ideas regarding a concept within the videos themselves, followed by a narrator (himself) explicitly addressing ideas generated during the interviews. However, the experience may be passive for the user who never interacts with the video other than via thought predictions. The IVVs, on the other hand, draw users in as they must answer multiple choice questions regarding physical events, and engage with video analysis or observe slow motion video, all in an effort to address naïve beliefs. Although directly engaging the user in Socratic dialogue is not possible, each IVV was scripted with the elicit, confront, resolve, reflect (ECRR) instructional framework in mind due to its demonstrated success in addressing misconceptions in physics $[7,10]$. This framework is related to Posner, Strike, Hewson, and Gertzog's Conceptual Change Model [4], and the ECRR technique has been found to yield positive results for student learning through face-to-face instruction $[11,12]$. However, the ECRR technique is less studied when applied to the design of instruction using web-based technologies.

Research on the effectiveness of the IVVs has found that some yield high learning gains, such as the Newton's Third Law IVV (N3 IVV), but the learning gains are much smaller for the Newton's Second Law IVV (N2 IVV), when compared with a control group that did not complete any IVVs [7]. The study reported in this paper was designed to explore how differences in student experiences with elements of the ECRR framework within both IVVs impacted any changes in their conceptions of Newton's Laws.

\section{METHODOLOGY}

An integrated mixed methods design was used in this study which combines the use of both quantitative and qualitative methods such that the phenomena under study is better understood than when using a single research method [13]. This design was chosen due to the need to collect both types of data simultaneously for each student. That is, the quantitative and qualitative strands affected the formation of each other where the integration of the data sets occurred during collection as well as analysis [14].

Nine undergraduate introductory physics students and twelve non-physics undergraduate students were recruited. Each group was randomly assigned to complete either the N2 
or N3 IVV, but seven students opted to complete both. As part of their participation, each student completed a short quantitative pre-test that contained questions regarding misconceptions about either Newton's Second (3 questions) or Third Law (5 questions). These questions were selected from the Force Concept Inventory [15], along with questions written by the LivePhoto Physics Group. All questions were carefully selected or designed to assess the learning outcomes of each IVV. After the pre-test, each student completed the related IVV and all interactions were recorded with a digital camcorder focused on the computer screen. This was followed by the student completing a quantitative post-test, which was identical to the pre-test and allowed for later quantitative analysis. Each student then participated in a qualitative video elicitation interview (VEI) which allowed the students to elaborate on their experience with the IVV while they watched a video of themselves completing it [16]. During this time the interviewer prompted the students to discuss their thinking or expand on why certain answer choices were selected for the questions in the IVV. The interviewer then discussed the pre- and post-test answers for each student, with a particular focus on those questions for which the student moved from an incorrect to a correct response. In these cases, the interviewer asked the student to explain where his or her thinking changed to determine how the IVV experience may have impacted their thinking.

A qualitative analysis of each interview was conducted such that instances of elicit, confront, resolve, or reflect were identified along with any changes in students' conceptions. A conceptual profile was created for each student such that initial conceptions, and any change in conceptions regarding Newton's Second or Third Law, could be tracked across the study, starting with the pre-test and continuing through the post-test and video elicitation interview. After individual conceptual profiles were created, common themes regarding student experiences with each IVV were determined.

\section{FINDINGS}

The thirteen N2 IVV study participants scored $15.4 \%$ on the N2 pre-test and $48.7 \%$ on the post-test for a medium Hake's gain of 0.39 [17]. The fifteen N3 study participants scored $25.3 \%$ on the N3 pre-test and $81.3 \%$ on the post-test for a high Hake's gain of 0.75 . In order to better understand the differences in gains, we provide detail here about how each IVV addressed the misconceptions assessed in these questions.

\section{A. Newton's Third Law IVV Findings}

The pre- and post-test questions for the N3 IVV assessed students' common naïve beliefs about Newton's Third Law including (1) greater mass implies greater force and (2) the most active agent produces the greater force [15]. The pretest results indicated that all students in the N3 group demonstrated both beliefs. However, only 4 of 15 students held onto the first conception after completing the IVV while 9 of 15 held onto the second conception.

It is interesting that the N3 IVV was more effective at improving student understanding of action/reaction forces when mass was considered, but not when differences in speed were involved, as both were targeted outcomes of the IVV. Upon analysis of the assessment data in conjunction with student interviews, it became apparent that a larger number of the students experienced the complete ECRR framework for conception (1), while no students experienced the framework for conception (2).

In order to better understand this, we provide a description of what students experience as they move through the N3 IVV. At the beginning, the student user observes video of passers-by on the street as they are interviewed by a narrator about the relative forces between two colliding carts of equal mass and speed. All predict that the forces would be equal. This is confirmed by the narrator who shows a video clip of the force versus time graph produced by force probes attached to the front of two colliding carts of equal mass. Student users are then asked to answer a multiple choice question about what they believe the relative collision forces will be between two cars of unequal mass and speed, a sedan and SUV. Based on student responses, this question tends to elicit conception (1) rather than (2). The former conception is then directly confronted in the IVV when the narrator shows a video clip of the force plot for the collision of two lab carts of unequal mass but similar speed, which clearly demonstrates the forces are equal and opposite. The passers-by in the IVV video are shown to be surprised by this outcome, much like the student user would be. Conversation with the narrator then serves to guide student users to resolve and reflect on their own understanding of these ideas, thereby providing users with the full ECRR experience as related to conception (1). Excerpts from one of the student interviews showcases what most N3 IVV participants experienced in terms of the ECRR sequence:

Interviewer: Ok, when she asked that question, what did you think the answer was?

El Rato (pseudonym): I thought the larger car would have the greater force exerted on the smaller car based on Newton's Second Law, considering that the mass was larger on the first, bigger car.

Interviewer: Describe for me your reactions to seeing the graph of the carts colliding?

El Rato: When I saw the graph, I knew I messed up. I didn't think the car that was heavier would have the same force exerted on it.

From the interview it is clear that El Rato believed conception (1). After watching a video of himself completing the IVV, El Rato explained that the video in the IVV, which showed a plot of the collision forces between the two cars as equal and opposite, convinced him that his initial conception was incorrect and that the forces would be equal. 
The student interviews within our study showed that the first multiple choice question in the N3 IVV elicited conception (1) from thirteen of the fifteen study participants. Twelve of these then experienced confront when shown the identical graphs of force versus time for the colliding carts. Eight later experienced resolve and reflect during the IVV. This means that more than half of the students that answered this question incorrectly at the beginning of the IVV experienced the full ECRR framework and changed their conceptions regarding conception (1). The success of moving these students to correct understanding demonstrates the power of the ECRR framework when applied to a single misconception in a short 7-minute web-based intervention.

On the other hand, the N3 IVV did not explicitly address conception (2). Although a question was posed during the IVV for the student to consider one car having a greater speed, the scenario also included that same car having a greater mass. During the interviews it was found that all students focused on differences in mass when answering the question and not a single student mentioned the relative speed of the two colliding cars. This indicates the need to explicitly address each targeted naïve belief.

\section{B. Newton's Second Law Findings}

For the N2 IVV, three alternate conceptions found in the literature were assessed on the pre- and post-test, or expressed by a participant spontaneously during the VEI, as related to learning outcomes of this IVV. These included: (A) force causes acceleration to terminal velocity, (B) velocity is proportional to applied force and (C) an active force eventually wears out [15]. As shown in Table I, most students demonstrated more than one of these.

TABLE I. The number of students (of 13) who exhibited a specific misconception at a given point within the study.

\begin{tabular}{lccc}
\hline \hline \multicolumn{1}{c}{ Misconception } & $\begin{array}{c}\text { Pre- } \\
\text { Test }\end{array}$ & $\begin{array}{c}\text { Post- } \\
\text { Test }\end{array}$ & VEI \\
\hline Force causes acceleration to & & & \\
terminal velocity & 5 & 4 & 5 \\
Active force wears out & 7 & 1 & 3 \\
$\begin{array}{l}\text { Velocity proportional to } \\
\text { applied force }\end{array}$ & 12 & 10 & 0 \\
\hline \hline
\end{tabular}

During the N2 IVV, students are presented with a scenario of a rider (narrator) sitting on a skateboard propelled by a fire extinguisher (constant force). The student user is asked to predict the motion of the skateboard by choosing one of five provided velocity versus time graphs (elicit), each designed around one of the expected misconceptions. The user then engages in video analysis of the recorded motion by clicking on the skateboard frame-by-frame to create a velocity versus time graph on the screen. The resulting plot is a line with positive slope (confront). This is then repeated for a fan cart videotaped in a lab setting where conditions are more controlled. This also sets the stage for the next segment in the IVV where users are asked to answer a multiple choice question to predict the acceleration of a cart with double the mass (elicit). A third video analysis is conducted with the heavier mass cart. At this point the user is asked to determine the slope of the line on the new velocity versus time graph and compare it to the slope that resulted from the lighter cart's video analysis. The differences in slope are obvious and the IVV ends with the narrator discussing how force is proportional to mass times acceleration. It is this part of the IVV which provides students the opportunity to resolve alternative conception(s) and reflect on how these ideas make sense in light of the graphing activities within the IVV.

In analyzing the student interviews along with the preand post-test data, it appeared that most students engaged with the ECRR framework for conception (C), but not for other conceptions. This may explain the success of this IVV in targeting this particular conception as seven of the thirteen students exhibited conception (C) on the pre-test, but only one maintained this belief on the post-test. Here we provide excerpts from a transcript involving the student whose misconception was not addressed by the N2 IVV, which seems to be due to her lack of understanding of other principles. The transcript begins after the student answers the first multiple choice question in the IVV in which she is asked to select the graph which describes the motion of the skateboard propelled by the fire extinguisher:

Interviewer: Now it seemed like you were doing a lot of thinking when selecting graph "b". What were your thoughts at this point? [Choice "b" was a graph related to conception (C).]

Clarissa (pseudonym): Um, I think I was looking at it as acceleration versus time, even though it says it was velocity over time.

Interviewer: So, how do you know whether your prediction is correct?

Clarissa: So my prediction is wrong.

Interviewer: Did you know then, looking between the two?

Clarissa: I don't think so.

In this instance, Clarissa's ideas were elicited but not confronted until she looked at the IVV for a second time during her interview. It was only then that she experienced confront but still could not decide which graph was correct. Here she also reflected on her understanding and knew that she had mixed up velocity with acceleration, but her lack of understanding was never resolved.

Although the data indicates that conception (C) was addressed by this IVV, the other two targeted conceptions were not. On the pre-test, twelve of thirteen students demonstrated conception (B) and only two were able to resolve this through the IVV. Although the graphs resulting from the video analyses were expected to have confronted this conception, it was observed during the interviews that many of the students, like Clarissa, did not have a clear understanding of velocity and acceleration graphs. This has led to additional IVVs be created to precede the N2 IVV and will hopefully address this issue. 
As for conception (A), the N2 IVV was not able to overcome it for the majority of students. Four of the five who demonstrated this conception on the pre-test continued to hold onto this belief on the post-test, while others demonstrated this incorrect understanding during the video elicitation interview (VEI). Again, it was expected that the velocity versus time graphs for a constant force would dispel this idea, but students' lack of understanding of velocity and acceleration may have impacted learning here as well.

\section{CONCLUSIONS}

Several findings from this research are important as they will inform future IVV development. Most important is how the ECRR framework was experienced by students as they completed each IVV. The ECRR experiences of El Rato (N3) and Clarissa (N2) were chosen for this paper because they showcase different ways the framework was experienced in light of specific alternative conceptions. El Rato experienced the ECRR framework in the N3 IVV in sequence and within moments of his naïve beliefs being elicited. His experience was similar to many others who completed the N3 IVV and had their alternative conceptions addressed. Clarissa, on the other hand, did not experience the full ECRR framework during the N2 IVV and never had her alternative ideas addressed, even during the VEI. Her outcome was similar to others who did not experience the full ECRR framework within either IVV. Both outcomes regarding student experiences with the ECRR framework and their ability to overcome certain alternative conceptions support the research literature on the effectiveness of the ECRR framework at promoting conceptual change [10].

In addition, we found that alternative conceptions were more often addressed for those explicitly targeted in the IVV and around which students experienced the entire ECRR framework. For example, the N3 IVV was successful at overcoming conception (1) but this was not the case for conception (2). Although this is not necessarily surprising, it does indicate the need to explicitly design IVV segments around each targeted misconception. An additional experiment in the N3 IVV involving a collision between two carts of equal mass but different speeds may have been enough to address the latter conception. On this note, the first multiple choice question in the N2 IVV was able to identify one of several alternative conceptions held by students. Although not possible at the time this IVV was developed, branching to an IVV segment that explicitly targeted the elicited misconception rather than taking all users through the same sequence may have been more effective at addressing the variety of misconceptions within this IVV.

And last, prior knowledge of the students appeared to impede learning, particularly for the N2 IVV in which student understanding of the differences between velocity and acceleration were essential. This is challenging when designing instruction for a web-based environment. Since this difficulty was observed for the N2 IVV, several other IVVs have been produced to address student understanding of velocity and acceleration as well as graphs that describe motion. Although not yet tested, these new IVVs will precede the N2 IVV and research will be conducted on the effectiveness of IVVs completed in sequence.

As more instructors rely on web-based instruction to support student learning, it becomes particularly important to understand how to apply what has been learned in physics education research to this new environment. This paper highlights just a few of our findings which will be applied as more IVVs are developed and will hopefully inform others working in this area as well.

\section{ACKNOWLEDGEMENTS}

The authors wish to thank Robert Teese, Priscilla Laws, and David Jackson of the LivePhoto Physics Group for allowing us access to the IVVs and for providing comments.
[1] E. F. Redish, in Proceedings of the Varenna Summer School, "Enrico Fermi" Course CLVI, edited by M. Vicentinni and E. F. Redish (IOS Press, Amsterdam, 2003), pp. 1-64.

[2] M. T. H. Chi, J. D. Slotta, and N. de Leeuw, Learn. Inst., 4, 1 (1994).

[3] A. A. di Sessa, in International Handbook of Research on Conceptual Change, edited by S. Vosniadou (Routledge, New York, 2008).

[4] G. J. Posner, et al., Sci. Ed., 66, 2 (1982).

[5] S. Vosniado, Learn. And Instr., 4, 1 (1994).

[6] L. C. McDermott and P. S. Shaffer, Tutorials in Introductory Physics, (Prentice Hall, Upper Saddle River, 1998).

[7] P. W. Laws, et al., Phys. Teach., 53, 2 (2015).

[8] compadre.org/ivv
[9] D. A. Muller, Ph. D. Thesis, University of Sydney, 2008.

[10] L. C. McDermott, Am. J. Phys., 69, 11 (2001).

[11] M. Allen, J. Res. Sci. Teach., 47, 2 (2010).

[12] K. Miller, et al, Phys. Rev. ST Phys. Educ. Res., 9, 020113 (2013).

[13] J. Creswell and V. L. Plano Clark, Designing and Conducting Mixed Methods Research, (Sage, Thousand Oaks, 2007).

[14] C. Teddlie and A. Tashakkori, Foundations of Mixed Methods Research, (Sage, Los Angeles, 2009).

[15]D. Hestenes, M. Wells, and G. Swackhamer, Phys. Teach., 30, 3 (1992).

[16] V. K. Otero and D. B. Harlow, in Getting Started in PER, Review in PER Vol. 2, edited by C. Henderson and K. A. Harper (AAPT, College Park, 2009).

[17] R. R. Hake, Am. J. Phys., 66, 1 (1998). 